\title{
Liposarcoma retroperitoneal gigante con compromiso renal izquierdo, reporte de un caso en el hospital "San Francisco de Quito"
}

\author{
Gabriel Medina Donoso ${ }^{1,2}$, Marcelo Cevallos Unda ${ }^{1}$, Verónica Alexandra Angos \\ Mediavilla $^{1,2}{ }^{\square}$ yiguel Moyón Constante ${ }^{1}$
}

1 Hospital San Francisco de Quito, Servicio de Cirugía General, IESS. Quito, Ecuador

2 Universidad Central del Ecuador, Postgrado de Cirugía General, ISP, Quito, Ecuador.

$\square:$ verito_angosm@outlook.com

Recibido: 2016-02-10; aceptado: 2016-04-28

\begin{abstract}
RESUMEN.- Los liposarcomas localizados en el retroperitoneo son por lo general lesiones de grado bajo e intermedio. La sintomatología predominante está relacionada con el efecto de masa. Dentro de los estudios de imagen, la tomografía simple y contrastada ha mostrado una adecuada sensibilidad. La terapia quirúrgica es la única opción que ofrece potencialmente beneficios curativos cuando se realiza resección completa con márgenes libres. El punto central de este estudio fue una paciente de 40 años, cuyo motivo de consulta fue prurito vaginal, dolor abdominal leve en hipogastrio y dispareunia tratada inicialmente por vaginosis a repetición. Al persistir dolor abdominal se solicita tomografía que evidencia masa hipodensa en región retroperitoneal que envuelve al riñón y al sistema pielocalicial del lado izquierdo. Se realizó laparotomía previa colocación de catéter doble J: el procedimiento fue excéresis de masa retroperitoneal más nefrectomía izquierda. Histopatología reportó liposarcoma de grado bajo con bordes de resección libres. Al momento, los controles tomográficos son negativos para recidivas. El tratamiento quirúrgico radical de los tumores de partes blandas ha mostrado un resultado favorable, en este caso, el resultado fue similar a los de estudios realizados de excéresis radical. La detección oportuna y el manejo multidisciplinario pueden ofrecer una terapia curativa.
\end{abstract}

PALABRAS CLAVES: compromiso renal, liposarcoma retroperitoneal, resección.

ABSTRACT.- Liposarcomas located in the retroperitoneum are usually low and intermediate grade lesions. The predominant symptoms are related to the mass effect. In imaging studies, with and without contrast tomography showed adequate sensitivity. Surgical therapy is the only therapy that offers potentially curative benefits when complete resection with clear margins is performed. The objective of this study was a female patient, 40 years, whose complaint was vaginal itching, mild abdominal pain at the level of umbilicus and dyspareunia, initially treated for recurrent vaginosis by persisting with abdominal pain tomography showed hypodense retroperitoneal mass region that surrounds the kidney and collecting system on the left side. Laparotomy was performed and double J stent placement, the procedure was exeresis more left nephrectomy retroperitoneal mass. Histopathology reported low grade liposarcoma with free resection edges. In this time, tomographic controls are negative for recurrences. Radical surgical treatment of soft tissue tumors has shown a favorable outcome. In this case, the outcome has been similar to those reported in studies of radical exeresis. Early detection and multidisciplinary management can provide a curative therapy.

KEYWORDS: renal commitment, resection, retroperitoneal liposarcoma.

\section{INTRODUCCIÓN}

Los sarcomas son tumores malignos que se originan del tejido conectivo tanto esquelético como extraesquelético, incluyendo al sistema nervioso periférico. La mayoría de estos tumores se localizan en los miembros (Mullen y DeLaney 2014); sin embargo, pueden localizarse en varias partes del cuerpo incluyendo el retroperitoneo; esta localización abarca entre el 10 al 15\% de todos los sarcomas de partes blandas (Raut y Pisters 2006).

En el ámbito nacional no se han reportado casos de sarcomas retroperitoneales en el registro de tumores del Instituto Nacional de Estadística y Censos (INEC). En el sitio web de la Sociedad de 
Lucha Contra el Cáncer del Ecuador (SOLCA) en el período 2005-2008 se reporta un total de 19 casos (8 en hombres y 11 en mujeres) diagnosticados como tumor maligno de peritoneo y retroperitoneo. CIE C-48 (SOLCA 2016).

Los liposarcomas localizados en el retroperitoneo son por lo general lesiones de grado bajo e intermedio y en su mayoría suelen ser bien diferenciados (grado bajo); tumores tipo mixoide y pleomórfico no suelen ser comunes (Lahat et al. 2009). Una característica de estos tumores es su baja capacidad para desarrollar metástasis a pesar que, su índice de recidiva suele ser elevado, sobre todo en aquellos que son mal diferenciados (Porter et al. 2006). En este grupo, la tasa de recurrencia es del 20 al 30\% en comparación con el $0 \%$ en los bien diferenciados; de igual manera, la mortalidad en los tumores mal diferenciados es 6 veces mayor (Gronchi et al. 2009).

Las manifestaciones clínicas se hallan relacionadas con la compresión y los efectos de masa que estos tumores suelen provocar a los distintos órganos adyacentes involucrados; muchos pacientes suelen ser diagnosticados de manera incidental al presentar sintomatología leve o incluso ser asintomáticos. Al momento del diagnóstico, el tamaño promedio suele ser de $15 \mathrm{~cm}$ (Mullen y DeLaney 2014). El efecto de masa como sintomatología predominante determina la presencia de edema de miembros inferiores y sintomatología de tipo neurológico por compresión de estructuras neurovasculares. A nivel gastrointestinal, los síntomas son saciedad precoz, obstrucción y en ocasiones sangrado; raramente se puede observar ascitis por compresión portal (Fiore et al. 2012).

Dado que en estos pacientes se debe descartar entidades como el linfoma, en la evaluación inicial se debe incluir la determinación de lactato deshidrogenasa (LDH) y de alfa fetoproteína; incluso se recomienda la determinación de gonadotropina coriónica humana ante sospecha de tumores de células germinales (Gronchi et al.2009). La tomografía simple y contrastada ha mostrado una adecuada sensibilidad para identificar las características y el compromiso de estructuras adyacentes (Ardoino et al. 2010). Los criterios de irresecabilidad están dados por los hallazgos de imagen e incluyen los siguientes: compromiso vascular intenso (grandes vasos), implantes peritoneales, metástasis a distancia, compromiso de la raíz del mesenterio y compromiso de la médula espinal (Ardoino et al. 2010) (Mullen y DeLaney 2014).

La resección completa con márgenes libres es la única opción quirúrgica que ofrece potenciales beneficios curativos, según el tiempo de evolución al momento de la cirugía (Singer et al. 2003) (Anaya et al. 2010). La quimio y radioterapia pre quirúrgica y como posterior terapia adyuvante no ha mostrado beneficios adicionales (Donahue et al. 2010) (Choi et al. 2012). Se recomienda un seguimiento tomográfico posterior a la resección con controles cada 3 a 6 meses durante los primeros 3 años. (Toulmonde et al. 2014).

Se presenta el caso de una paciente adulta sin antecedentes de importancia que inicia con sintomatología ginecológica tratada inicialmente como micosis vaginal; al no obtener mejoría y añadirse metrorragias se continuó la investigación con exámenes complementarios; destacan los hallazgos tomográficos de masa hipodensa de aproximadamente $45 \times 11 \times 28 \mathrm{~cm}$ en la región retroperitoneal que envuelve al riñón y al sistema pielocalicial del lado izquierdo. Se decide realizar laparotomía más excéresis de la masa, previa consulta con urología, para colocación de catéter doble J que facilite la identificación del uréter. La paciente presenta evolución postquirúrgica favorable por lo que se decide el alta hospitalaria al sexto día posterior al procedimiento. Se realizó control en consulta externa a los 5 días del alta, se retira drenaje. El estudio histopatológico del espécimen reportó liposarcoma bien diferenciado, con márgenes tumorales libres, sin signos de invasión linfovascular.

Se considera que la revisión bibliográfica de esta entidad poco habitual es de suma importancia para el diagnóstico diferencial de masas en retroperitoneo, aportando al proceso formativo de médicos cirujanos. Al tratarse de una patología inusual, el reporte de su manejo clínico y quirúrgico en diferentes instituciones aporta al mejoramiento e innovación de los protocolos de manejo, con un beneficio potencial para los pacientes involucrados.

\section{CASO CLÍNICO}

Se trata de una paciente de 40 años de edad, empleada privada, nacida y residente en Quito, de estado civil casada, de instrucción superior, sin antecedentes clínicos ni quirúrgicos de importancia; su motivo de consulta fue prurito vaginal, dolor abdominal de leve intensidad a nivel de hipogastrio y antecedente de dispareunia de aproximadamente 2 años de evolución, acompañada de secreción vaginal fétida en moderada cantidad. El diagnóstico inicial del servicio de ginecología fue: micosis vaginal; recibió tratamiento medicamentoso sin resultados. 
Acude nuevamente a consulta con ginecología por presentar varios episodios recurrentes; adicionalmente refiere metrorragias de evolución más reciente por lo que, se indica realización de eco abdominal y pélvico sin evidencia de patología alguna por lo que, se solicita tomografía abdominopélvica (Figura 1A y 1B) en la cual se aprecia masa hipodensa de aproximadamente $45 \times 11 \times 28 \mathrm{~cm}$ en la región retroperitoneal que envuelve al riñón y al sistema pielocalicial del lado izquierdo, razón por la cual es referida al servicio de cirugía general de esta casa de salud.

Signos vitales.- FC: 60 lpm; FR:16 rpm; TA: 100 / 60 T: 36.2; Peso: 75.9Kg; Talla: $156 \mathrm{~cm}$ e IMC: 31

Examen físico de abdomen.- blando, depresible, con masa palpable de aproximadamente $40 \mathrm{~cm}$ en mesogastrio, hipogastrio flanco y fosa iliaca izquierda, de consistencia blanda, no dolorosa, sin adenomegalias palpables.

Como parte del diagnóstico diferencial se tomó en cuenta como posibilidades: linfoma, tumores primarios de células germinales, masas duodenales, pancreáticas, renales y suprarrenales (Mullen y DeLaney 2014), cuando se localizan en la línea media deben incluir como diagnóstico diferencial a los "schwannomas" y paragangliomas (Lawrence et al. 1987); sin embargo, la imagen tomográfica, la morfología y las características hipodensas de la misma junto con su ubicación fueron altamente sugestivas de una neoplasia tipo sarcoma, probablemente liposarcoma retroperitoneal gigante.

Biometría Hemática.- Leucocitos: 7.63 (5.0-10.0), Neutrófilos: $54.8 \%$ (55\%-65\%), Linfocitos: $34.4 \%$ (10\%-30\%), Hemoglobina 15.7g/dL (12-16g/dL), Hematocrito: $45.9 \%(40 \%-50 \%)$, Plaquetas 223000 (100 000-350 000).

Tiempos de Coagulación.- TP: 12.4 (10-15); INR: 1.05(0.8-1.2); TTP: 33 (20-40).

Química Sanguínea.- Amilasa : 72.0 UI/1 (50-120 $\mathrm{UI} / 1)$; Creatinina: $0.79 \mathrm{mg} / \mathrm{dL}(0.4-1 \mathrm{mg} / \mathrm{dL})$; Glucosa 91.9mg/dL (60-110mg/dL); LDH: 196 (120-200); Lipasa: $53.0 \mathrm{U} / \mathrm{L}(50-150 \mathrm{U} / \mathrm{L})$.

Por la densidad y la localización de la masa, determinadas por el estudio tomográfico, se diagnóstica un tumor retroperitoneal compatible con liposarcoma. Se decide realizar laparotomía más excéresis de la masa previa consulta con urología para colocación de catéter doble J que facilite la identificación del uréter (Figura 1A y 1B) (Figuras 2A y 2B).

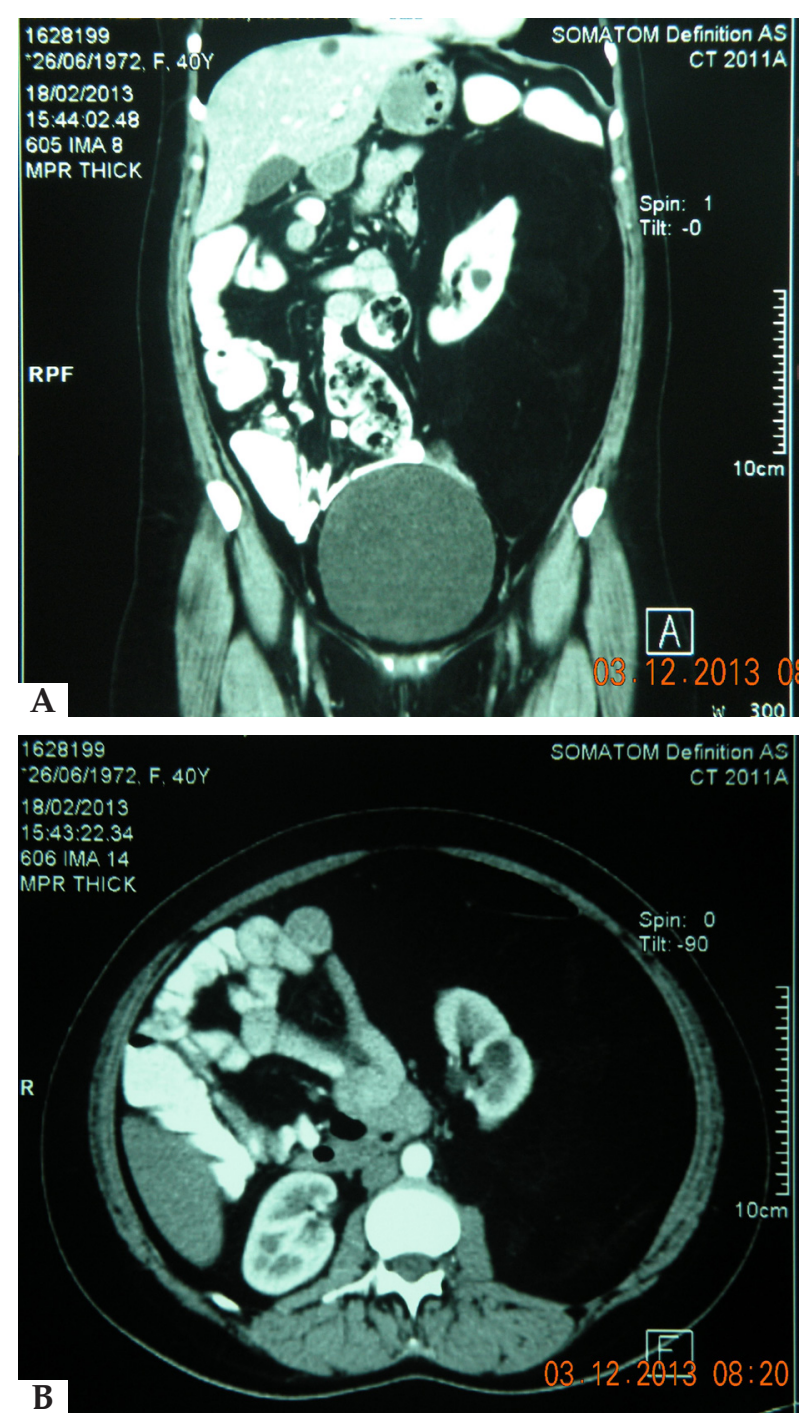

Figura 1. Estudio tomográfico contrastado: A) plano transversal y B) plano coronal. Tumoración englobando el riñón izquierdo y delimitada por la fascia perirrenal se observa una masa homogénea, con UH que varían entre -86 y -100. (Densidades de grasa), desplaza a todas las estructuras intraabdominales hacia la derecha, incluyendo al riñón izquierdo, el cual se encuentra centralizado
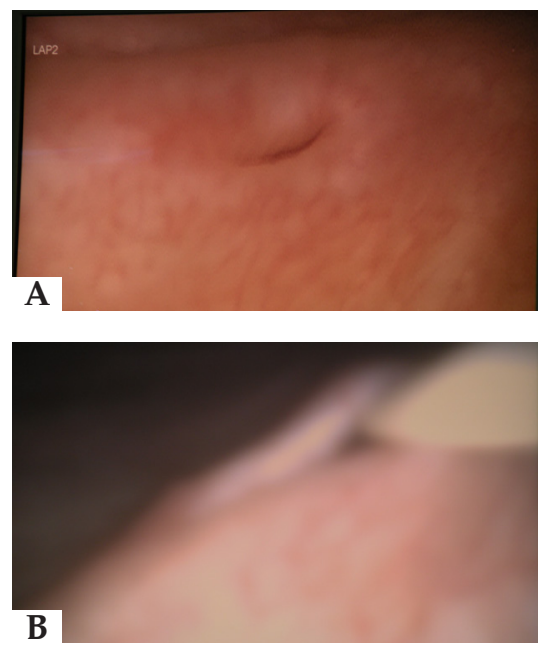

Figura 2. Cistoscopía: A) Identificación de meato ureteral izquierdo mediante cistoscopia y B) canalización de uréter izquierdo con catéter doble J 


\section{EVOLUCIÓN}

Se realiza laparotomía con abordaje suprainfraumbilical con los siguientes hallazgos: tumor retroperitoneal adherido a la pared

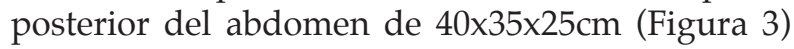
que compromete riñón e hilio renal izquierdo en $\mathrm{su}$ interior sin evidencia de compromiso visceral ni de planos osteo-musculares adyacentes; no se encuentran siembras de tumor compatibles con metástasis en cavidad. Se realiza excéresis de masa retroperitoneal que se halla adherida a la fascia lumbar y a la cola del páncreas y que envuelve completamente al riñón izquierdo sin ofrecer un plano de clivaje adecuado (Figuras 4A y 4B), por lo cual se decide realizar una nefrectomía izquierda colocándose un drenaje aspirativo en la zona de disección entre el plano posterior del tumor con la fascia lumbar y la cola del páncreas. El uréter izquierdo se identificó mediante la palpación del catéter previamente colocado (Figura 5).

La paciente presenta evolución postquirúrgica favorable, fue manejada en terapia intensiva durante las 72 horas posoperatorias iniciales. Los valores de hemoglobina de $9 \mathrm{mg} / \mathrm{dl}$ y hematócrito de $28 \%$ determinan la administración de 2 paquetes globulares con control posterior de hemoglobina de $11 \mathrm{mg} / \mathrm{dl}$ y hematocrito de $34 \%$. Se obtiene tolerancia oral adecuada al cuarto día, con adecuado manejo del dolor; se decide el alta hospitalaria al sexto día posterior al procedimiento. Se realizó control en consulta externa a los 5 días del alta retirándose el drenaje. El estudio histopatológico reportó liposarcoma bien diferenciado, con márgenes tumorales libres, sin signos de invasión linfovascular.

La cirugía se realizó en abril de 2013, el primer control posoperatorio de lo realiza a los diez días evidenciándose una adecuada evolución; en este control se retira drenaje. El control posterior se efectúa a los tres meses (julio de 2013), luego a los seis meses (octubre de 2013) se realiza un control tomográfico sin evidencia de recidiva. Los dos últimos controles se los realiza al año de la cirugía (abril de 2014 y abril de 2015) sin evidencia de recidiva y con una adecuada evolución y calidad de vida.

El reporte de este caso se lo realizó con la autorización del jefe de servicio de Cirugía General del hospital San Francisco de Quito y se mantuvo durante todo el proceso de la realización de este trabajo la confidencialidad de la paciente.

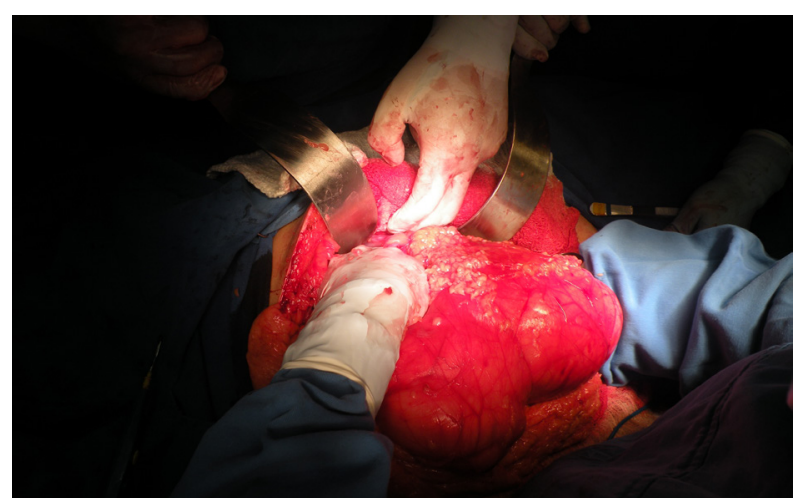

Figura 3. Hallazgo transquirúrgico: gran tumoración retroperitoneal de aspecto lipomatoso

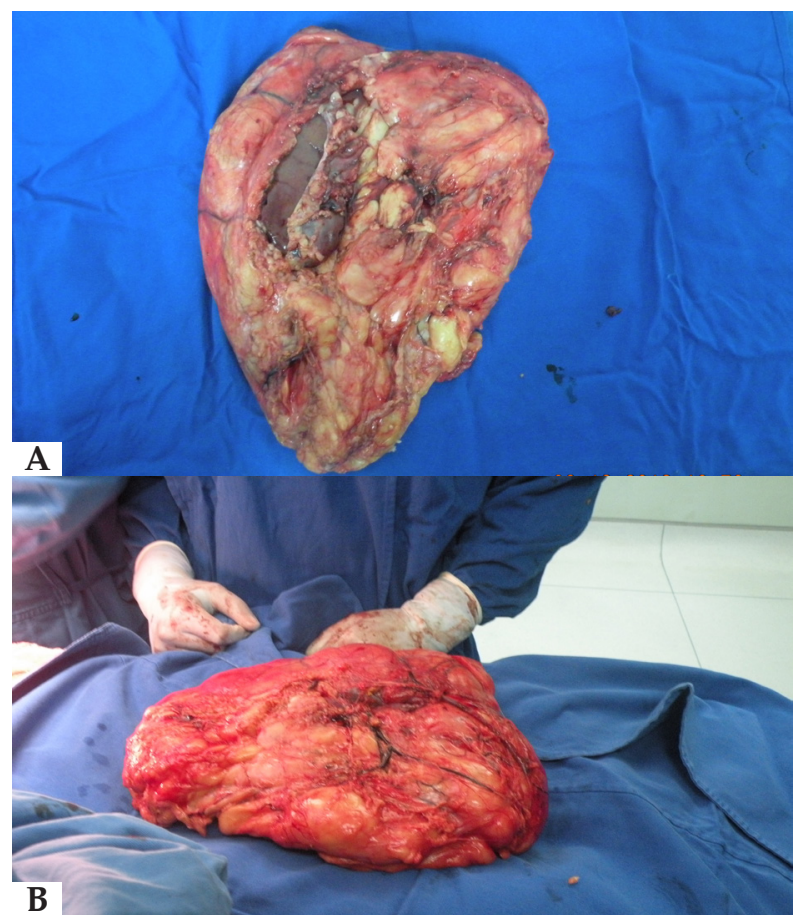

Figura 4. Pieza anatómica: A) Vista anterior de tumoración y B) vista interna de Tumoración, se distingue el compromiso renal

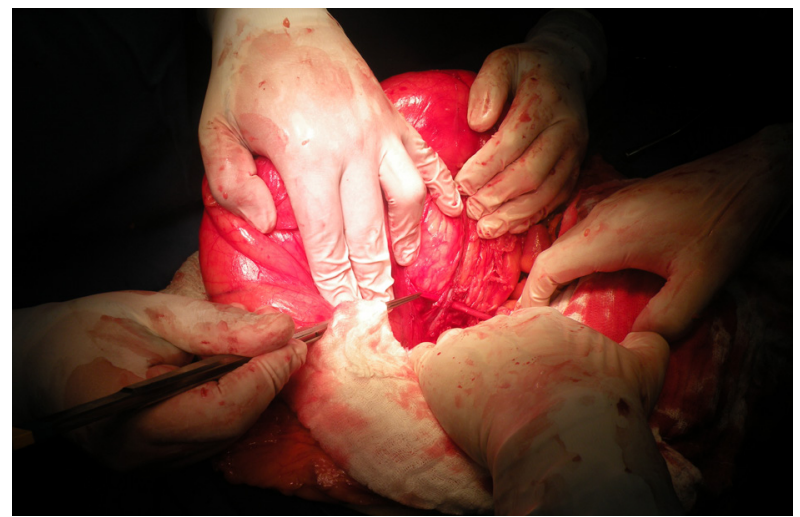

Figura 5. Identificación de uréter izquierdo que se introduce en masa tumoral 


\section{DISCUSIÓN}

El caso presentado fue el primero de esas características tratado en el hospital San Francisco de Quito. Esta descripción coincide con el reporte de incidencia anual de liposarcomas de aproximadamente 2.7 casos por millón de personas, sin que exista diferencias de aparición respecto del sexo de los pacientes afectados (Mullen y DeLaney 2014).

Como referencias importantes se menciona que aproximadamente el $80 \%$ de los sarcomas localizados en el retroperitoneo suelen ser malignos, el subtipo histológico más común son los liposarcomas y los leiomiosarcomas (Evans 2007). Otros autores indican que la conformación histológica de los liposarcomas comprende células como adipocitos, lipoblastos que contienen núcleos atípicos y con varias vacuolas grasas intracitoplasmáticas, suelen contener además un infiltrado de células inflamatorias (Mullen y DeLaney 2014). En el caso descrito el estudio histopatológico reportó liposarcoma bien diferenciado, con márgenes tumorales libres, sin signos de invasión linfovascular.

El abordaje de este tipo de tumores implica la necesidad de un estudio completo para definir la etiología del mismo (Lahat et al. 2008). En el caso que se reporta, la presentación clínica insidiosa se asocia a la dificultad diagnóstica inherente; pocos fueron los síntomas relacionados con el efecto de masa que provocó el tumor en la paciente, se trató en sí de un hallazgo incidental.

Lahat y colaboradores (2008) describen que la resección completa de la masa tumoral se obtiene en el 50 al 67\% de casos (Lahat et al. 2009). En ocasiones es necesaria la excéresis junto con el tumor de órganos comprometidos, en orden de frecuencia: riñón, colon, bazo, y páncreas, lo cual indica que se requiere un control óptimo acerca de la función renal antes de la cirugía. En este caso una vez definido el diagnóstico se identificó además el compromiso renal del lado izquierdo, lo cual obligó a una evaluación de la función renal la misma que se no se encontró alterada. El motivo de la colocación de catéter doble J fue para ayudar en la identificación del uréter durante la laparotomía ya que el objetivo inicial fue la preservación del riñón izquierdo; sin embargo y como es evidente, en las imágenes se tomó la decisión de realizar le excéresis de la masa junto con la nefrectomía izquierda al no existir un adecuado plano clivaje.

De acuerdo con la bibliografía citada cuando la imagen obtenida en estudios tomográficos muestra un patrón típico que sugiera liposarcoma los estudios de biopsias o punción no muestran utilidad (Mullen y DeLaney 2014). Esta recomendación se realizó en este caso ya que en base a los hallazgos de imagen se decidió el tratamiento.

La evolución de la paciente fue favorable y el resultado final de histopatología demuestra los beneficios de la terapia quirúrgica radical para el pronóstico vital.

\section{CONCLUSIONES}

El tratamiento quirúrgico de excéresis radical de los tumores de partes blandas ha mostrado resultados favorables en las series reportadas; el resultado de este caso ha sido similar a lo reportado.

La presentación clínica insidiosa es un factor importante a considerar, ya que, como en un alto porcentaje de procesos neoplásicos, su diagnóstico se realiza en estadíos avanzados lo cual, evidentemente, dificulta la posibilidad de ofrecer al paciente una terapia curativa. El enfoque multidisciplinario es fundamental para el proceso diagnóstico, la preparación prequirúrgica, y el soporte posquirúrgico inmediato y tardío.

Es necesario realizar el seguimiento de este caso a largo plazo y obtener nuevos casos para profundizar la comprensión integral de esta entidad e identificar factores asociados y nuevos esquemas de manejo en consonancia con los avances del conocimiento existente.

\section{REFERENCIAS BIBLIOGRÁFICAS}

Anaya D, Lahat G. y Wang X. 2010. Postoperative nomogram for survival of patients with retroperitoneal sarcoma treated with curative intent. Annals of Oncology 21.

Ardoino I, Miceli R y Berselli M. 2010. Histologyspecific nomogram for primary retroperitoneal soft tissue sarcoma. Cancer $\mathbf{1 1 6 .}$

Choi A, Barnholtz-Sloan J y Kim J. 2012. Effect of radiation therapy on survival in surgically resected retroperitoneal sarcoma: a propensity score-adjusted SEER analysis. Annals of Oncology 23.

Donahue T, Kattan M. y Nelson S. 2010. Evaluation of neoadjuvant therapy and histopathologic response in primary, high-grade retroperitoneal sarcomas using the sarcoma nomogram. Cancer 116. 
Evans H, 2007. Atypical lipomatous tumor, its variants, and its combined forms: a study of 61 cases, with a minimum follow-up of 10 years. The American Journal of Surgical Pathology 31.

Fiore M., Colombo C y Locati P. 2012. Surgical technique, morbidity, and outcome of primary retroperitoneal sarcoma involving inferior vena cava. Annals of Surgical Oncology 19.

Gronchi A, Lo Vullo S, Fiore M y et al, 2009. Aggressive surgical policies in a retrospectively reviewed single-institution case series of retroperitoneal soft tissue sarcoma patients. Journal of Clinical Oncology 27.

Lahat G, Madewell J y Anaya D. 2009. Computed tomography scan-driven selection of treatment for retroperitoneal liposarcoma histologic subtypes. Cancer 115(108).

Lahat G, Tuvin D y Wei C. 2008. New perspectives for staging and prognosis in soft tissue sarcoma. Annals of Surgical Oncology 15.

Lawrence W, Donegan W y Natarajan N. 1987. Adult soft tissue sarcomas. A pattern of care survey of the American College of Surgeons. Annals of Surgery 205.

Mullen J y DeLaney T. 2014. Clinical features, evaluation, and treatment of retroperitoneal soft tissue sarcoma. Página de Internet: www. uptodate.com

Porter G, Baxter N y Pisters P. 2006. Retroperitoneal sarcoma: a population-based analysis of epidemiology, surgery, and radiotherapy. Cancer 106(7).

Raut C y Pisters P. 2006. Retroperitoneal sarcomas: Combined-modality treatment approaches. Journal of Surgical Oncology 94(81).

Singer S, Antonescu C, Riedel E y Brennan M.. 2003. Histologic subtype and margin of resection predict pattern of recurrence and survival for retroperitoneal liposarcoma. Annals of Surgery 238.

SOLCA, 2016. Registro de Tumores SOLCA matriz. Página de Internet: http://www.estadisticas. med.ec/webpages / reportes / Tablas1-2.jsp. Consultada 25-abril- 2016.

Toulmonde M, Le Cesne A y Mendiboure J. 2014. Long-term recurrence of soft tissue sarcomas: Prognostic factors and implications for prolonged follow-up. Cancer $\mathbf{1 2 0}$ 